\title{
Turismo sustentável e objetivos de desenvolvimento sustentável: perspectiva bibliométrica avaliativa e relacional no período 2015- 2020
}

O artigo teve como objetivo analisar a produção científica internacional do turismo sustentável dentro da perspectiva dos Objetivos de Desenvolvimento Sustentável - ODS, visando compreender as características das publicações científicas. A metodologia utilizada foi de abordagem exploratória-descritiva, o procedimento consistiu em análise de artigos referentes as temáticas de Turismo Sustentável e ODS, a partir da base de dados Web of Science abrangendo o período de 2015 a 2020, com a palavra-chave usada 'Sustainable tourism'; 'sustainable development goals', foram executadas duas etapas de filtragem. Na primeira, optou-se por manter como tipos de documentos artigos e revisões e, na segunda, procedeu-se uma exclusão de áreas de pesquisa fora do escopo da temática em estudo, totalizando, ao final, 232 artigos. A etapa seguinte constitui-se de avaliação relacionada ao ano de publicação, áreas de pesquisa, periódicos, países, idioma, autores e número de citação. O uso do software VosViewer possibilitou a organização de grupos (cluster) baseados em cocitação e as redes de coocorrências de palavras-chave, em uma perspectiva qualitativa. Como resultados, observou-se que a pesquisa sobre essa temática se encontra em um estágio inicial da discussão, com um crescimento no volume de artigos publicados no período de 2015 a 2020 . Os termos mais destacados giram em torno das palavraschave turismo sustentável, turismo, desenvolvimento sustentável e objetivos de desenvolvimento sustentável, ou seja, as elencadas como foco do estudo. No tocante aos cluster de coautoria, os resultados apontam para formações numerosas de clusters de autores (206) ainda que, seja perceptível a dispersão nas redes sinalizando, aparentemente, a jovialidade da temática ODS.

\section{Sustainable tourism and sustainable development objectives: evaluative and relational bibliometric perspective in the 2015-2020 period}

\begin{abstract}
The article aimed to analyze the international scientific production of sustainable tourism within the perspective of the Sustainable Development Goals - SDGs, to understand the characteristics of scientific publications. The methodology used was the qualitative, exploratory-descriptive approach, or the procedure composed in analyzes of articles as themes of Sustainable Tourism and SDGs, from the Web of Science database, covering the period from 2015 to 2020 , with the keywordkey used 'Sustainable tourism'; 'sustainable development objectives', two filtering steps were performed. In the first, choose to keep articles and analysis as types of documents and, in the second, proceed with the exclusion of research areas outside the scope of the subject under study, totaling, in the end, 232 articles. One step to follow, to assess the publication's performance, research areas, journals, countries, language, authors and citation number. The use of the VosViewer software enabled the organization of groups (cluster) based on cocitation and as networks of co-occurrences of keywords, from a qualitative perspective. As a result, use research on this topic and find an early stage of the discussion, with an increase in the volume of articles published in the period from 2015 to 2020. The most prominent terms will be displayed around the keywords of sustainable tourism, tourism, sustainable development, and sustainable development objectives, that is, as listed as the focus of the study. Regarding the co-authorship cluster, the results pointed to numbered formations of groups of authors (206) although, the dispersion in the networks is noticeable, apparently signaling the youthfulness of the SDG theme.
\end{abstract}

Keywords: Sustainable Tourism; Sustainable Development Goals; Bibliometric Study.

Topic: Desenvolvimento, Sustentabilidade e Meio Ambiente

Reviewed anonymously in the process of blind peer

Lúcia Silva Albuquerque de Melo (it)

Universidade Federal de Campina Grande, Brasil

http://lattes.cnpq.br/7135220151360318

http://orcid.org/0000-0002-9543-5642

luciasalbuquerque@gmail.com

Maria de Fátima Nóbrega Barbosa (iD

Universidade Federal de Campina Grande, Brasil

http://lattes.cnpq.br/8262057016878547

http://orcid.org/0000-0003-3415-8829

mfnobregabarbosa@gmail.com
Received: $12 / 05 / 2020$

Approved: 05/06/2020
Referencing this:

MELO, L. S. A.; BARBOSA, M. F. N.. Turismo sustentável e objetivos de desenvolvimento sustentável: perspectiva bibliométrica avaliativa e relacional no período 2015-2020. Revista Ibero-Americana de Ciências Ambientais, v.11, n.4, p.371-385, 2020. DOI: http://doi.org/10.6008/CBPC2179-6858.2020.004.0030 


\section{INTRODUÇÃO}

As Organizações das Nações Unidas (ONU) definiu uma série de metas por meio dos Objetivos de Desenvolvimento Sustentável (ODS) para acabar com a pobreza, proteger o meio ambiente e o planeta, bem como assegurar a prosperidade para todas as nações, conhecidos como Agenda 2030 (HALL, 2019). Esses objetivos buscam integrar de modo equilibrado as dimensões econômica, social e ambiental do desenvolvimento que causam impactos na sociedade e no meio ambiente, dentre os quais se insere a atividade turística.

Dada a ênfase da Organização Mundial do Turismo (OMT), sobre turismo sustentável e a importância econômica do setor, os Objetivos de Desenvolvimento Sustentável tornaram-se pontos focais para estudar o tema, contribuindo para o setor (SAARINEN et al., 2014; ROBU et al., 2019). Viagens e turismo têm contribuição significativa para o moderno sistema econômico. De acordo com as estatísticas relatadas pela OMT, a indústria global de viagens e turismo representou $9,8 \%$ do produto interno bruto global total e $7 \%$ do comércio global, em 2016. O setor também gerou aproximadamente $11 \%$ do emprego existente no mundo (direto e indireto) em 2016 (UNWTO, 2017). No Brasil, o turismo contribui com 8,5\% do PIB e gera 7 milhões de empregos (UNWTO, 2017).

No entanto, embora o turismo produza benefícios econômicos, esta contribuição para o desenvolvimento econômico também pode ter um impacto significativo para a degradação ambiental, impactos sociais e culturais negativos e a fragmentação de habitats. O crescimento não planejado do turismo prejudicou os ambientes naturais e socioculturais de muitos destinos turísticos (CHOI et al., 2006; PAN et al., 2018), e em muitos casos está gerando o fenômeno conhecido como overtourism, ou seja, o excesso de turistas frente a capacidade local. Os impactos negativos do turismo em um destino e aos seus stakeholders influenciam na qualidade de vida dos cidadãos e/ou qualidade das experiências dos visitantes de uma forma negativa (UNWTO, 2018). Nesse sentido, o crescimento do overtourism chama a atenção para a necessidade do planejamento sustentável da atividade turística (KOENS et al., 2018), para evitar a insustentabilidade da atividade, bem como, as reações de turismofobia, isto é, movimentos anti-turistas (SERAPHIN et al., 2018).

Esses efeitos colaterais indesejáveis levaram à crescente preocupação com a conservação e preservação de recursos naturais, o bem-estar humano e a viabilidade econômica a longo prazo das comunidades (HARALAMBOPOULOS et al., 1996; BUTLER, 1999; JACKSON, 2008; TANG, 2015) que são diretamente afetadas pelas atividades de turismo.

Dentro deste contexto, também se encontram as novas demandas da sociedade em termos de preservação do meio ambiente, justiça social, crescimento econômico, desenvolvimento territorial que agora são alavancas para ofertas turísticas exitosas, baseados em modalidades turísticas alternativas, como contraponto ao turismo de massa (MOLZ, 2013). Atualmente, alguns turistas buscam ser usuários responsáveis, pois adotam os princípios da justiça social e econômica em respeito ao meio ambiente e às culturas; reconhece a centralidade da comunidade local de acolhimento e o seu direito a ser protagonista no desenvolvimento do turismo sustentável e socialmente responsável pelo seu território; opera de acordo com 
o impacto de seu turismo no meio ambiente, reduzindo assim os impactos negativos ao meio ambiente (IMMACOLATA, 2018).

O turismo sustentável é um dos principais focos do debate sobre o desenvolvimento do turismo ambientalmente integrado e é uma modalidade baseada nos princípios do desenvolvimento sustentável (BUTLER, 1999; ETE, 2009; NUNKOO et al., 2011; ESLAMI et al., 2018; PAN et al., 2018).

Portanto, o crescente reconhecimento do papel do turismo sustentável no desenvolvimento da sustentabilidade e a conquista da Agenda de Desenvolvimento 2030 possibilitaram um avanço histórico que oferece uma oportunidade única para várias organizações intergovernamentais, governos nacionais, assim como para autoridades regionais e locais, comunidades, organizações empresariais e outros atores, instituições acadêmicas e organizações da sociedade civil de vários tipos, de desenvolverem e usarem os conjuntos de indicadores de sustentabilidade (HAK et al., 2012).

Diante desse contexto, este artigo tem como objetivo analisar a produção científica internacional do turismo sustentável dentro da perspectiva dos ODS, de modo a permitir a compreensão das características das publicações científicas. Para isso, foi utilizado técnicas avaliativas (medidas de produtividade e métricas de impacto) e técnicas relacionais (coautoria e coocorrência), tendo como parâmetros os principais autores, áreas das publicações, periódicos mais influentes, países e idiomas.

Por intermédio do software VOSviewer também foram geradas as redes com os clusters de coautoria e as palavras-chave com maior relação e frequência nas publicações sobre a temática. A análise foi realizada através de conteúdo disponível na base de dados Web of Science no período de 2015 a 2020, o recorte temporal admite-se em virtude da agenda 2030 ter sido implantada com os Objetivos de Desenvolvimento Sustentável a partir de 2015. Nesse sentido, a presente pesquisa se justifica pela relevância em disponibilizar uma visão abrangente do panorama atual da pesquisa sobre Turismo Sustentável e os Objetivos do Desenvolvimento Sustentável.

O presente estudo se configura a partir desta introdução, em uma seção de fundamentos teóricos acerca do Turismo Sustentável e os Objetivos de Desenvolvimento Sustentável, seguida por uma terceira seção para metodologia, para que na quarta seção siga a apresentação e discussão dos resultados, para finalmente, apresentar as conclusões do estudo, incluindo as limitações e indicações de estudos futuros.

\section{REVISÃO TEÓRICA}

O turismo é uma atividade humana que depende tanto dos recursos naturais quanto contribui para o seu esgotamento. Essa inter-relação pode ser direta ou indireta, e embora todas as atividades turísticas possam ser inevitavelmente locais, elas somam-se a fenômenos de importância global (GÖSSLING, 2002).

Nesse sentido, os impactos globais do turismo estão ganhando maior atenção da sociedade. As mudanças climáticas, a urbanização costeira, a perda da biodiversidade, o consumo de combustíveis fósseis, a transmissão de doenças e a comoditização cultural estão entre as questões turísticas mais controversas que permeiam a mídia. No entanto, até recentemente, os impactos econômicos positivos do turismo foram o foco principal, com muito menos ênfase nas consequências ambientais e sociais (RUTTY et al., 2015; SCOTT 
et al., 2019).

O turismo sustentável tornou-se foco de atenção através de uma combinação de fatores. 0 crescimento do turismo internacional e o aumento do foco no ambientalismo na sociedade levaram a uma maior conscientização sobre o significado dos princípios da sustentabilidade. Dessa forma, a sustentabilidade tornou-se uma narrativa dominante dentro do desenvolvimento da política turística em todos os níveis territoriais (BAUM et al., 2016; MILLER et al., 2010).

Contudo, no setor turístico existe um discurso de uso da sustentabilidade e das comunidades locais, por parte dos defensores desse setor, para fortalecer suas bases de poder e legitimar práticas insustentáveis atuais, em vez de trabalhar genuinamente para alcançar os benefícios de uma abordagem sustentável (BUCKLEY, 2012; MAXIM, 2015). Nessa perspectiva, o equilíbrio dos interesses socioeconômicos com os sistemas ecológicos finitos do planeta é um objetivo fundamental da sustentabilidade. Embora os danos ambientais significativos continuem, tem havido um impulso e progresso em direção a caminhos de sustentabilidade no turismo (BOLUK et al., 2019).

A Figura 1 mostra um roteiro para conectar o setor do turismo ao desenvolvimento sustentável em todo o mundo. Desde o final dos anos 80 , a pesquisa no campo do turismo tem dado foco substancial à questão da sustentabilidade (ou desenvolvimento sustentável) (HALL, 2010). Naquela época, foi proposto e desenvolvido o conceito de ligação entre a atividade turística e o meio ambiente, conhecido como turismo sustentável (AALL, 2014).

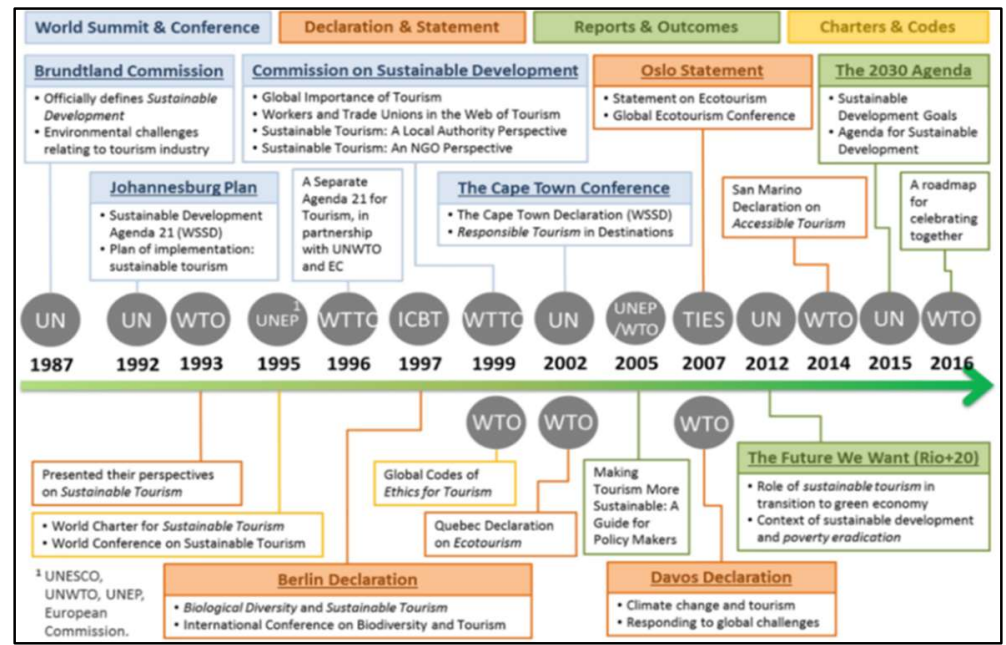

Figura 1: Roteiro que conecta o turismo ao desenvolvimento sustentável em todo o mundo: princípios, declarações, convenções, declarações e códigos. Fonte: Pan et al. (2018).

O plano de ação das Nações Unidas (ONU) para a 'Agenda 21 de Desenvolvimento Sustentável' adotado na Conferência do Rio em 1992. Depois disso, a Organização Mundial do Turismo apresentou suas perspectivas sobre o turismo sustentável. Em 1996, foi publicada uma Agenda 21 separada para o turismo, desenvolvida em parceria com a Organização Mundial do Turismo (UNWTO), Conselho Mundial de Viagens e Turismo (WTTC) e o Conselho da Terra (CE). Seguida por este documento, a Declaração de Quebec sobre "Ecoturismo" foi anunciada na Cúpula Mundial de Ecoturismo em 2002, e a Declaração de Davos sobre "Mudança climática e turismo respondendo aos desafios globais" foi anunciada posteriormente em 2007. 
Até a Rio + 20 em 2012, o documento final "O futuro que queremos" promoveu amplamente o papel do turismo na transição para uma economia verde no contexto do desenvolvimento sustentável e da erradicação da pobreza. Atualmente, no entanto, os interesses no turismo sustentável entre os pesquisadores ainda são limitados, embora o turismo esteja entre inúmeras partes interessadas, como os defensores do setor do turismo, empresas e turistas (BUCKLEY, 2012).

Um dos principais resultados da Conferência Rio +20 foi o acordo dos Estados membros de lançar um processo para desenvolver uma agenda global, chamada Agenda 2030, e um conjunto de Objetivos de Desenvolvimento Sustentável - ODS (SANTOS et al., 2015). Os ODS foram formulados através de um exaustivo e participativo processo de discussão por diversos grupos de interesse em todo o mundo, incluindo a sociedade civil organizada, setor privado e órgãos locais (KLOPP et al., 2017).

Embora não seja juridicamente vinculativa, a Agenda 2030 da ONU pretende ser o ponto de referência para o desenvolvimento internacional, exortando os governos a traduzir os ODS globais em metas e políticas nacionais, a formular uma visão clara e de longo prazo do desenvolvimento nacional (LUCAS et al., 2016) e definir metas, indicadores e prioridades específicas de cada país, dependendo das condições e capacidades nacionais (WEITZ et al., 2015). Contudo, os governos precisam estar cientes desse processo, considerando que existem muitos desafios para promover o desenvolvimento sustentável.

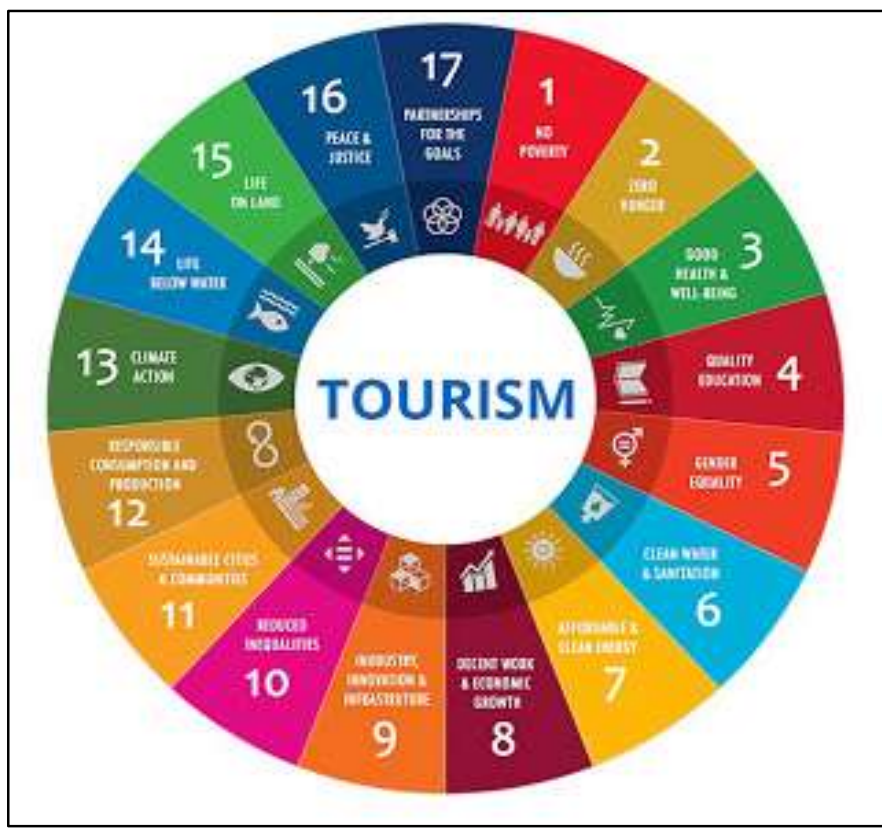

Figura 2: Integração Turismo e ODS. Fonte: UNWTO (2017).

$\mathrm{Na}$ lógica dos ODS, os 17 objetivos individuais representam os diversos elementos da sustentabilidade e que, como conjunto, eles fornecem uma representação holística da complexidade e interdependências do desenvolvimento sustentável. Cada objetivo tem um número de metas e indicadores associados. Como uma agenda globalmente acordada, os ODS compreendem um conjunto de objetivos ambiciosos e complexos, sem precedentes, objetivos que compreendem uma estrutura de monitoramento por meio de relatórios anuais à ONU (VALENCIA et al., 2019). Para melhor compreensão, segue Figura 2 com a integração dos 17 ODS com o Turismo. 
Dentro da perspectiva da Agenda 2030, a Organização Mundial do Turismo argumentou que o turismo tem o potencial de contribuir para atingir todos os ODS, direta ou indiretamente. Assim, o turismo está intimamente ligado aos ODS (BOLUK et al., 2017; SCHEYVENS, 2018) e recomendou cinco pilares centrais para os quais o turismo deve contribuir de forma significativa e duradoura para alcançar o desenvolvimento sustentável (UNWTO, 2017), a saber: (i) Crescimento econômico inclusivo e sustentável, que está ligado aos ODS 8, 9, 10 e 17; (ii) Inclusão social, emprego e redução da pobreza, que está ligado aos ODS 1, 3, 4, 5, 8 e 10; (iii) uso mais eficiente dos recursos naturais e proteção do meio ambiente, como indicam os ODS 6, 7, 8, $11,12,13,14$ e 15; (iv) valores culturais, diversidade e patrimônio, associados aos ODS 8, 11 e 12; e (v) compreensão mútua, paz e segurança, associadas aos ODS 16 (PAN et al., 2018).

Portanto, a transformação do turismo em direção à sustentabilidade exige uma abordagem interdisciplinar para sua implementação, como políticas integrativas (internacionais, nacionais e locais), investimentos em tecnologias inovadoras, fortes parcerias entre governos e empresas de turismo e promoção de práticas sustentáveis (por exemplo, uso eficiente de energia e água e gestão integrada de resíduos). A incorporação da educação ambiental para aumentar a conscientização do público também facilitaria nesse processo de transformação rumo à sustentabilidade

Para analisar a efetiva contribuição das atividades turísticas para o alcance dos ODS torna-se necessária, uma abordagem holística, devido às inter-relações entre os recursos naturais e culturais e as partes interessadas. Em resumo, para assegurar o desenvolvimento do turismo de forma sustentável, ele deve ser ecologicamente responsável, socialmente compatível, culturalmente apropriada, politicamente equitativo, tecnologicamente favorável e, finalmente, economicamente viável para a comunidade anfitriã (CHOl et al., 2006).

\section{METODOLOGIA}

Com o propósito de mapear a literatura internacional sobre Turismo sustentável e Objetivos do Desenvolvimento sustentável, a presente pesquisa possui abordagem exploratória-descritiva e se baseou na análise bibliométrica, com a finalidade de construir mapas científicos com base em dados bibliográficos. Muitos estudos foram realizados na análise e visualização de muitos tipos diferentes de redes bibliométricas, essas análises, muitas vezes, concentraram-se em redes de pesquisadores, redes de coautoria, palavraschave que ocorrem em publicações, dentre outras informações. (VAN ECK et al., 2010; VAN ECK et al., 2014). Portanto, essa análise bibliométrica possibilita o estudo do desenvolvimento de um determinado campo de pesquisa, bem como o delineamento da literatura sobre a temática pesquisada.

A base de dados utilizada nesta pesquisa foi a Web of Science (WOS), coleção principal. A Web of Science representa um dos mais relevantes indexadores de periódicos científicos, por sua abrangência, credibilidade e possibilidade de uso em diferentes softwares de análise bibliométrica (GLÄNZEL, 2003; LUCAS et al., 2015, QUEIROZ et al., 2017). A pesquisa foi realizada em 06 de fevereiro de 2020 na base de dados e teve como recorte temporal o período de 2015 a 2020, em virtude da implantação da agenda 2030 e dos 
Objetivos de Desenvolvimento Sustentável ter acontecido a partir de 2015. Para a adequada organização do estudo, foi desenvolvido um protocolo de pesquisa para o delineamento do estudo, conforme ilustrado na figura 3.

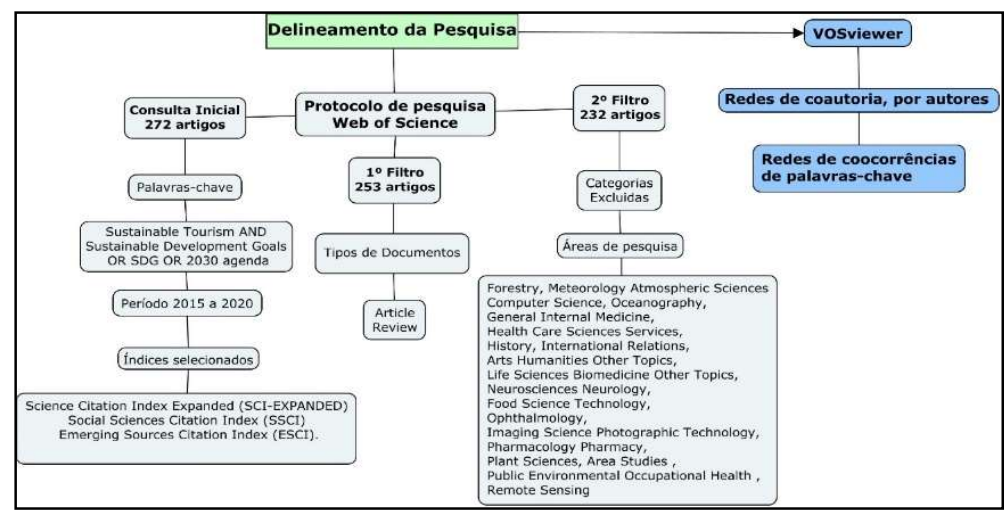

Figura 3: Procedimento para delineamento da Pesquisa.

O Protocolo de pesquisa tomou como plano de busca, a consulta inicial com foco nas palavras-chave 'Sustainable tourism and sustainable development goals or SDG or 2030 agenda', resultando em um total de 272 artigos localizados. Na sequência, foram executadas duas etapas de filtragem. No primeiro filtro, optouse por manter como tipos de documentos artigos e revisões e, na segunda, procedeu-se uma exclusão de áreas de pesquisa fora do escopo da temática em estudo, totalizando, ao final, 232 artigos.

Posteriormente, o resultado foi exportado atendendo ao critério de 'salvar em outros formatos de arquivo', gravando-se o conteúdo de 'registro completo e referências citadas' no formato de arquivo 'texto sem formatação' em um formato de arquivo separado por tabulações, tendo como finalidade a utilização dos dados no software VOSViewer para construção das análises bibliométricas.

A etapa seguinte constitui-se de avaliação relacionada ao ano de publicação, áreas de pesquisa, periódicos, países, idioma, autores e número de citação. O uso do software VosViewer possibilitou a organização de grupos (cluster) baseados em cocitação e as redes de coocorrências de palavras-chave, em uma perspectiva qualitativa.

\section{RESULTADOS E DISCUSSÃO}

Nesta seção são apresentados e comentados os resultados obtidos nas análises bibliométricas realizadas. Os indicadores bibliométricos utilizados foram os seguintes: número de documentos publicados por ano; países onde os artigos foram publicados; áreas das publicações investigadas, periódicos em que os autores publicaram pesquisas, idioma de publicação de pesquisas, autores e obras mais citados nos artigos pesquisados, bem como as palavras-chave mais frequentes nos artigos pesquisados.

Propondo-se a analisar o período de 2015 a 2020 da produção científica internacional do turismo sustentável dentro da perspectiva dos ODS, o estudo identificou 232 publicações indexadas na base Web of Science (WOS), disponibilizadas até a data de consulta realizada em 06 de fevereiro de 2020. A soma do número de citações foi 930, uma média de 4,01 citações por publicação. 


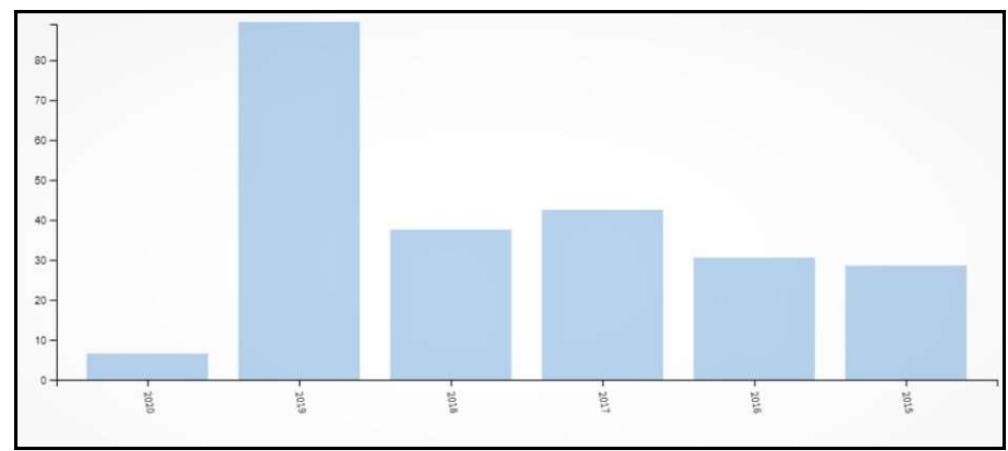

Figura 4: Quantidade de publicações por ano.

Verifica-se um número mais significativo de publicações no ano de 2019, com 89 publicações, em comparação com os anos de 2015 a 2018, perfazendo 38\% do total da amostra, no qual uma tendência de crescimento se mostra presente. Tendo em vista que a temática sobre ODS é recente, surgindo a partir de 2015, naturalmente o nível de publicação nos primeiros anos será menor.

No tocante às áreas das publicações investigadas, a área com mais publicações é Social Sciences Other Topics, seguida por Environmental Sciences Ecology e Science Technology Other Topics, no qual as três comportam mais de $80 \%$ das publicações localizadas. No que se refere aos períodicos foram identificados entres as 232 publicações um total de 118 periódicos. Quando analisa-se os periódicos mais influentes, observa-se que em número de registros o destaque é para o periódico Sustainability que alcançou um total de 36 (15\%) publicações, seguido pelo Journal of Sustainable Tourism com 31 (13\%) publicações , assim como os periodicos Tourism Management Perspectives e Tourism Management que tiveram, respectivamente, $6 \mathrm{e}$ 5, a mairoria dos períodicos apresentou apenas uma (1) publicação.

Com relação a frequência de publicações por país, identificou um total de 75 países que desenvolveram algum tipo de estudo direcionado a temática do Turismo Sustentável e ODS. Entre os 10 países com maior número de publicações os Estados Unidos é o país que mais se destaca por apresentar o maior número de registros (30), seguido da Austrália (24), Espanha (19), China (18), Canada (16), Inglaterra (15), Nova Zelândia (13), Taiwan (12), Polônia (11) e Romênia (11). Os 10 países em conjunto respondem por aproximadamente $72 \%$ de todas as publicações da temática estudada.

Tabela 1: Relação dos 10 dos autores com maior número de publicações.

\begin{tabular}{|l|l|l|l|}
\hline Ordem & Autores & Documentos & Número de citações \\
\hline 1 & Higgins-Desbiolles, F. & 5 & 46 \\
\hline 2 & Baum, T. & 4 & 51 \\
\hline 3 & Cavaliere, C. & 3 & 13 \\
\hline 4 & Gossling, S. & 3 & 18 \\
\hline 5 & Hall, C. & 3 & 28 \\
\hline 6 & Scheyvens, R. & 3 & 85 \\
\hline 7 & Wu, T. & 2 & 65 \\
\hline 8 & Adshead, D. & 2 & 7 \\
\hline 9 & Boley, B. & 2 & 30 \\
\hline 10 & Boluk, K. & 2 & 3 \\
\hline
\end{tabular}

Com referência ao idioma, optou-se na pesquisa por pesquisar em todas as línguas, buscando com isso uma abrangência maior de publicações, visto que a temática pesquisada é relativamente nova, foram identificadas das 232 publicações, 216 são de origem inglesa representando 93\%, seguido do idioma 
espanhol (11), português (2), russo (2) e italiano (1). Quanto aos autores, foram elencados os dez autores em números de produções e citações, entre as 232 identificadas na pesquisa, como apresentado na tabela 1.

Observa-se na tabela 1 que os autores que se destacam no ranking dos artigos mais citados, Scheyvens e Wu, não são os que possuem maior número de publicações na área pesquisada. Com base nos temas-chave delimitados no estudo, foram ranqueados os trabalhos que apresentam maior relevância, listados em função do seu impacto, quanto ao critério de citação, utilizou-se o Fator H (H-index) disponibilizado na Web of Science, que no campo estudado possui um Fator $\mathrm{H}$ igual a 15, conforme dados apresentados no quadro 1.

Quadro 1: Fator H (H-Index) das publicações investigadas.

\begin{tabular}{|c|c|c|c|c|}
\hline Título & Autores & Título da Fonte & Ano & Citações \\
\hline $\begin{array}{l}\text { The Private Sector and the SDGs: The Need to } \\
\text { Move Beyond 'Business as Usual' }\end{array}$ & $\begin{array}{l}\text { Scheyvens, Regina; Banks, } \\
\text { Glenn; Hughes, Emma }\end{array}$ & $\begin{array}{l}\text { Sustainable } \\
\text { Development }\end{array}$ & 2016 & 58 \\
\hline $\begin{array}{l}\text { Community sustainability and resilience: } \\
\text { similarities, differences and indicators }\end{array}$ & 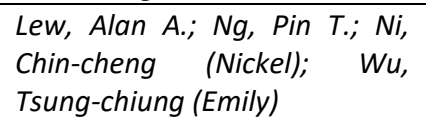 & $\begin{array}{l}\text { Tourism } \\
\text { Geographies }\end{array}$ & 2015 & 42 \\
\hline $\begin{array}{l}\text { Politics and sustainable tourism: The case of } \\
\text { Cyprus }\end{array}$ & $\begin{array}{l}\text { Farmaki, Anna; Altinay, Levent; } \\
\text { Botterill, David; Hilke, Sarina }\end{array}$ & $\begin{array}{l}\text { Tourism } \\
\text { Management }\end{array}$ & 2015 & 34 \\
\hline $\begin{array}{l}\text { Sustainability and the Tourism and Hospitality } \\
\text { Workforce: A Thematic Analysis }\end{array}$ & $\begin{array}{l}\text { Baum, Tom; Cheung, } \\
\text { Catherine; Kong, Haiyan; Kralj, } \\
\text { Anna; Mooney, Shelagh; Hai } \\
\text { Nguyen Thi Thanh; } \\
\text { Ramachandran, Sridar; Ruzic, } \\
\text { Marinela Dropulic; Siow, May } \\
\text { Ling }\end{array}$ & Sustainability & 2016 & 32 \\
\hline $\begin{array}{l}\text { Sustainable tourism: Sustaining tourism or } \\
\text { something more? }\end{array}$ & Higgins-Desbiolles, Freya & $\begin{array}{l}\text { Tourism } \\
\text { Management } \\
\text { Perspectives }\end{array}$ & 2018 & 30 \\
\hline $\begin{array}{l}\text { Tourism Entrepreneurship Performance: The } \\
\text { Effects of Place Identity, Self-Efficacy, and } \\
\text { Gender }\end{array}$ & $\begin{array}{l}\text { Hallak, Rob; Assaker, Guy; Lee, } \\
\text { Craig }\end{array}$ & $\begin{array}{l}\text { Journal of Travel } \\
\text { Research }\end{array}$ & 2015 & 25 \\
\hline $\begin{array}{l}\text { Moving beyond sense of place to care of place: } \\
\text { the role of Indigenous values and } \\
\text { interpretation in promoting transformative } \\
\text { change in tourists' place images and personal } \\
\text { values }\end{array}$ & $\begin{array}{l}\text { Walker, Kaye; Moscardo, } \\
\text { Gianna }\end{array}$ & $\begin{array}{l}\text { Journal of } \\
\text { Sustainable } \\
\text { Tourism }\end{array}$ & 2016 & 24 \\
\hline Inclusive tourism development & $\begin{array}{l}\text { Scheyvens, Regina; Biddulph, } \\
\text { Robin }\end{array}$ & $\begin{array}{l}\text { Tourism } \\
\text { Geographies }\end{array}$ & 2018 & 22 \\
\hline $\begin{array}{l}\text { Empowerment and resident support for } \\
\text { tourism in rural Central and Eastern Europe } \\
\text { (CEE): the case of Pomerania, Poland }\end{array}$ & $\begin{array}{l}\text { Strzelecka, Marianna; Boley, B. } \\
\text { Bynum; Strzelecka, Celina }\end{array}$ & $\begin{array}{l}\text { Journal of } \\
\text { Sustainable } \\
\text { Tourism }\end{array}$ & 2017 & 20 \\
\hline $\begin{array}{l}\text { Weighing and realizing the environmental, } \\
\text { economic and social goals of tourism } \\
\text { development using an analytic network } \\
\text { process-goal programming approach }\end{array}$ & Zhang, Jiekuan & $\begin{array}{l}\text { Journal of Cleaner } \\
\text { Production }\end{array}$ & 2016 & 19 \\
\hline $\begin{array}{l}\text { Constructing sustainable tourism } \\
\text { development: The } 2030 \text { agenda and the } \\
\text { managerial ecology of sustainable tourism }\end{array}$ & Hall, C. Michael & $\begin{array}{l}\text { Journal } \\
\text { Sustainable } \\
\text { Tourism }\end{array}$ & 2019 & 17 \\
\hline $\begin{array}{l}\text { Performance Indicator Framework for } \\
\text { Evaluation of Sustainable Tourism in the } \\
\text { Taiwan Coastal Zone }\end{array}$ & $\begin{array}{l}\text { Wang, Shih-Hao; Lee, Meng- } \\
\text { Tsung; Chateau, Pierre- } \\
\text { Alexandre; Chang, Yang-Chi }\end{array}$ & Sustainability & 2016 & 17 \\
\hline $\begin{array}{l}\text { The environmental management problem of } \\
\text { Pohorje, Slovenia: A new group approach } \\
\text { within ANP-SWOT framework }\end{array}$ & $\begin{array}{l}\text { Groselj, Petra; Stirn, Lidija } \\
\text { Zadnik }\end{array}$ & $\begin{array}{l}\text { Journal of } \\
\text { Environmental } \\
\text { Management }\end{array}$ & 2015 & 17 \\
\hline $\begin{array}{l}\text { Sustainable human resource management as } \\
\text { a driver in tourism policy and planning: a } \\
\text { serious sin of omission? }\end{array}$ & Baum, Tom & $\begin{array}{l}\text { Journal of } \\
\text { Sustainable } \\
\text { Tourism }\end{array}$ & 2018 & 15 \\
\hline 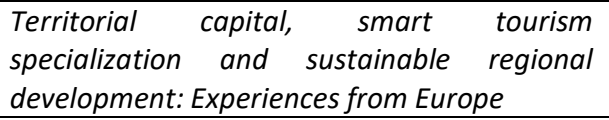 & Romao, Joao; Neuts, Bait & $\begin{array}{l}\text { Habitat } \\
\text { International }\end{array}$ & 2017 & 15 \\
\hline
\end{tabular}


Observa-se, no quadro 1, que a obra com maior impacto é The Private Sector and the SDGs: The Need to Move Beyond 'Business as Usual', o referido estudo de Scheyvens et al. (2016) apresenta uma revisão da literatura acerca do desenvolvimento dos ODS, a fim de explorar o papel do setor privado na definição da agenda de desenvolvimento pós-2015, os tipos de mecanismo de desenvolvimento postulados para o setor privado e o potencial que isso representa no tocante a melhorias sociais, econômicas e resultados ambientais. $\mathrm{O}$ estudo realiza uma discussão e reflexão crítica sobre o que pode e deve ser esperado de empresas que, distintas das organizações governamentais e da sociedade civil, têm uma razão de ser centrada na obtenção de lucro, refletindo, portanto, sobre o potencial do setor privado de contribuir para práticas mais sustentáveis e responsáveis e sobre os limites desse processo.

O artigo "Community sustainability and resilience: similarities, differences and indicators" (LEW et al., (2015) é o segundo artigo mais citado do ranking, a pesquisa apresenta e discuti um quadro conceitual para esclarecer as semelhanças e diferenças entre sustentabilidade e resiliência, focando em suas principais diferenças de conservação e adaptação. Com base nessas definições, são apresentados indicadores em uma escala de desenvolvimento comunitário, derivados de pesquisas em comunidades orientadas ao turismo na zona rural de Taiwan.

O terceiro artigo mais citado, "Politics and sustainable tourism: The case of Cyprus", cujos autores são Farmaki et al. (2015), foi citado 34 vezes. O estudo traz uma discussão dos mecanismos que podem viabilizar e restringir a implementação do turismo sustentável e incorpora conceitos-chave da literatura existente, bem como investiga os fatores políticos que influenciam a implementação do turismo sustentável no Chipre.

A pesquisa de Baum et al. (2016) explora o posicionamento da força de trabalho dentro da discussão sobre o turismo sustentável, ou a ausência dele, e, em seguida, busca demonstrar a relevância da narrativa da sustentabilidade para uma seleção de mão-de-obra relacionada à força de trabalho no contexto do turismo e da Agenda 2030 de Desenvolvimento Sustentável das Nações Unidas. Os autores enfatizam que, apesar da negligência existente com a força de trabalho, defendem que o emprego e a força de trabalho do turismo estejam no centro da narrativa do turismo sustentável.

Higgins-Desbiolles (2018) argumenta na sua pesquisa que o turismo deve ser compreendido e gerido num contexto mais amplo de sustentabilidade. Além disso, as abordagens estratégicas para a transição para uma abordagem suficiente do turismo e do lazer são essenciais para garantir a sustentabilidade.

Complementando a pesquisa e a demonstração gráfica de dados bibliográficos, foi utilizado o software Vosviewer ${ }^{\circledR}$ version 1.6.12, com a finalidade de mapear as relações e clusters formados por publicações e autores que compõem o universo de dados estudado.

A figura 5 apresenta o Mapa da rede de relacionamento dos autores com agrupamento baseado em cocitação. A Análise de Cocitação, mensura a vinculação entre dois artigos com base no número de publicações em que estes aparecem citados conjuntamente. Portanto, a cocitação identifica a associação de dois documentos citados, via suas frequências de ocorrência conjunta em uma lista de referências dos 
autores citantes (GRÁCIO, 2016). Na visão de Small (1973) ao se medir a força de cocitação entre dois documentos, se demonstra o grau de associação entre pares de documentos, segundo a compreensão da comunidade de autores citantes.

A rede de coautoria evidencia os relacionamentos entre os autores mais citados e sua relação com base nas publicações produzidas, com a intenção de que quanto mais citado o autor é, quanto maior ele é representado. Seu agrupamento ocorre unindo as obras que se referem um ao outro, apresentando clusters com base em temas relacionados de publicação que produz trabalhos sobre o tema e cocitação entre eles.

Outro aspecto que merece destaque, em virtude da natureza exploratória deste estudo, foi a inclusão de todos os autores com alguma publicação no escopo da pesquisa, dado que somente 22 do total de 656 autores possui mais de uma publicação. Consequentemente, elaborar a rede com apenas os 22 autores limitariam a análise inicial da temática, assim como sua descrição.

Foram identificados 656 autores tratando da problemática em estudo. Muitos destes, conforme se observa na figura 5, não pertencem fortemente a nenhum cluster ou pertencem a clusters de tamanhos reduzidos. Isso se explica pela pouca maturidade do campo de estudo, visto que os trabalhos científicos foram publicados a partir de 2015. Um total de 206 clusters foram identificados com 1076 links entre autores. Os principais clusters de cooperação se encontram circulados na figura 5.

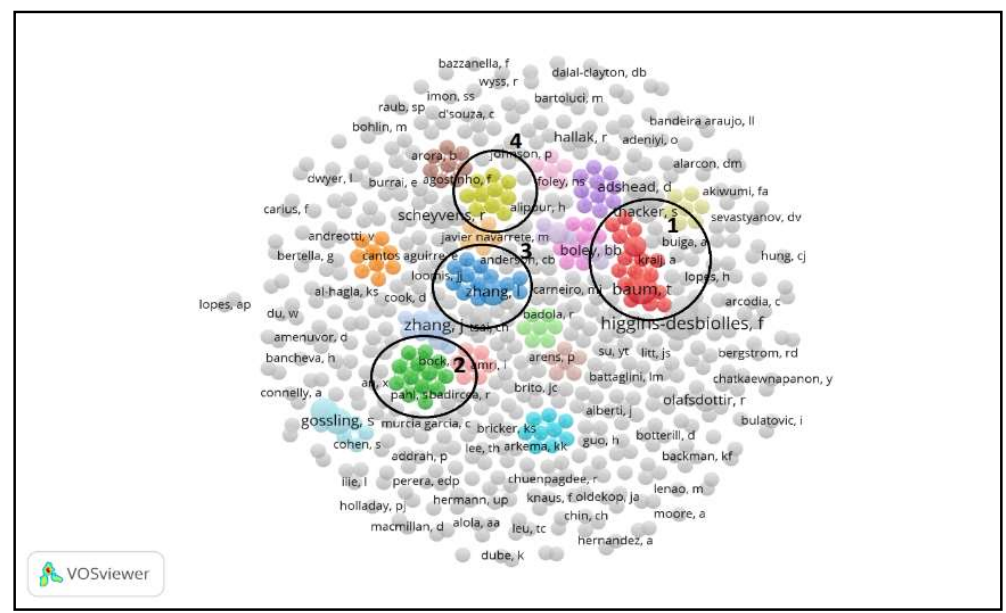

Figura 5: Rede de coautoria, por autores.

O principal cluster é formado por 17 autores (número 1 em vermelho). Neste cluster o principal expoente é Baum com 4 publicações e uma relação de força tamanho 12. Em seguida, aparece Bu com 1 publicações relação de força tamanho 4. Também é destaque Cheung. C com 1 publicações relação de força tamanho 8. 0 segundo cluster de destaque é composto por 13 autores (número 2 em verde). A maioria dos autores possui apenas uma publicação e relação de força tamanho 12 . O terceiro cluster de destaque é composto por 13 autores (número $3 \mathrm{em}$ azul). Nenhum autor possui mais que um trabalho publicado. Neste caso, todos os autores têm relação de força tamanho 9, com exceção para Zhang que possui 3 publicações e relação de força tamanho 13 . Observa-se ainda que os demais clusters apresentam o mesmo padrão do cluster 4, ou seja, apresentam apenas uma publicação cada autor e a mesma relação de força tamanho dentro do cluster. Outro aspecto verificado é o decréscimo na relação de força tamanho à medida que vai 
diminuindo o tamanho dos clusters.

O mapa de Palavras-Chave (Figura 6) com maior relação e frequência, apresenta a coocorrência de palavras, isto é, palavras que são reportadas em conjunto nas obras publicadas. Nesse sentido, Van Eck et al. (2014) relata que a relação de coocorrência entre duas palavras-chave é definida pelo número de publicações em uma base de documentos em que ambas ocorrem conjuntamente, seja no título, no resumo ou na lista de palavras-chave. Portanto, a proximidade de dois autores identificada por sua alta frequência de cocitação pode ser originária de várias possibilidades, entre elas: compartilhamento de referencial teórico e/ou metodológico, similaridade e/ou complementaridade de conteúdos e oposição teórico e/ou metodológica, entre outras (GRÁCIO, 2016).

No estudo foram selecionados termos presentes no título e abstract das 232 publicações da base de dados. Como critério de elaboração foram selecionados apenas os termos com, ao menos, dez menções, 1.401 foram considerados pelo software, e estes foram reduzidos a 19 itens de acordo com o grau de significância. Os resultados são demonstrados na Figura 6.

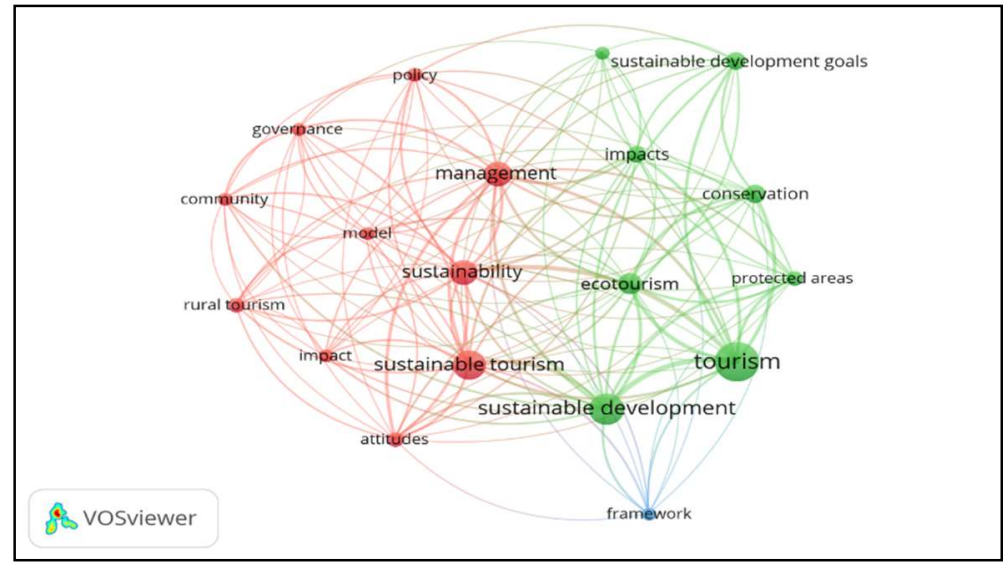

Figura 6: Rede de coocorrências de palavras-chave.

A figura 6 demonstra a existência de 2 conglomerados de termos. Tourism (turismo) é a palavrachave com a maior ocorrência, aparece 69 vezes como é evidenciado no mapa acima. O primeiro clusters em vermelho é composto por 10 termos. Sua estruturação está centrada em torno do Sustainable Tourism (Turismo Sustentável). Entre os termos presentes, os seguintes devem ser destacados por apresentar maior frequência de ocorrência são eles: sustainability (sustentabilidade), management (gestão), impacts (impactos), governance (governança), policy (política) rural tourism (turismo rural) e Community (comunidade).

O segundo cluster, representado pela cor verde, composto por 8 termos e sua composição está centrada em torno do turismo e desenvolvimento sustentável. Temos como sustainable development goals (objetivos de desenvolvimento sustentável) ecotourism (ecoturismo) conservation (conservação) protected áreas (áreas protegidas). No mapa é possível observar os elos entre as palavras incluídas nos clusters existentes, e este conjunto sugere pesquisas que abordam as relações entre o turismo e o desenvolvimento sustentável. A análise dos termos mais frequentes é relevante, especialmente, para campos de investigação novos, pois oferece insights sobre os principais interesses dos pesquisadores. Dessa forma, pode-se inferir 
que as temáticas, turismo sustentável e ODS, possui uma correlação forte entre elas.

\section{CONCLUSÕES}

O presente trabalho teve por objetivo analisar a produção científica internacional do turismo sustentável dentro da perspectiva dos ODS. Portanto, este estudo apresenta os resultados de um mapeamento e análise de artigos indexados na base Web of Science - Coleção Principal (Clarivate Analytics) que associam as temáticas, turismo sustentável e objetivos de desenvolvimento sustentável, os quais estão publicados principalmente nos periódicos Sustainability, Journal of Sustainable Tourism, Tourism Management Perspectives e Tourism Management. Observou-se que a pesquisa sobre essa temática se encontra em um estágio inicial da discussão, com um crescimento no volume de artigos publicados no período de 2015 a 2020.

As instituições mais influentes na pesquisa em turismo sustentável e ODS estão concentradas em países como Estados Unidos, Austrália, Espanha, China, Canada, Inglaterra e Nova Zelândia, sendo estes destaques na produção sobre a temática. A caracterização do campo de estudo, via utilização do software VosViewer, permitiu, através da elaboração de redes bibliométricas, concluir quais são os principais clusters de coautoria, entre autores, assim como quais os termos em evidência nas publicações sobre a temática.

No tocante aos cluster de coautoria, entre autores identificou-se quatro com maior relevância, no qual os principais autores são: Baum, Bu, Cheung, Zhang. Na sequência foi gerado o mapa com as palavraschave com maior quantidade de coocorrências, de forma que foi possível visualizar dois cluster, o primeiro centrado em torno do Sustainable Tourism (Turismo Sustentável), com destaque para as palavras-chaves: sustainability (sustentabilidade), management (gestão), impacts (impactos) e governance (governança). E o segundo com sua composição centralizada em volta do turismo e desenvolvimento sustentável, tendo como termos mais evidentes sustainable development goals (objetivos do desenvolvimento sustentável) ecotourism (ecoturismo) conservation (conservação) e protected áreas (áreas protegidas.

Considerando as características deste estudo e que, principalmente, a pesquisa se restringiu apenas aos dados da base Web of Science, o que pode ser apontado como uma limitação. Pois, apesar desta base ser considerada uma das mais relevantes, sugere-se que outros trabalhos sejam realizados em outras bases de dados, tais como Scopus, Science Direct, EBSCO, Scielo e periódicos específicos.

Em suma, este estudo ofereceu como contribuição um olhar genérico do cenário atual sobre turismo sustentável e ODS, mostrando a emergência e relevância do tema e como este vem sendo explorado. Tendo como objetivo apontar as características da produção científica que vem sendo desenvolvida nessa área, podendo servir de subsídio para pesquisas futuras.

\section{REFERÊNCIAS}

AALL, C.. Sustainable tourism in practice: Promoting or perverting the quest for a sustainable development? Sustainability (Switzerland), v.6, n.5, p.2562-2583, 2014. DOI: http://doi.org/10.3390/su6052562
BUCKLEY, R.. Sustainable tourism: Research and reality. Annals of Tourism Research, v.39, n.2, p.528-546, 2012. DOI: https://doi.org/10.1016/j.annals.2012.02.003 
BAUM, T.; CHEUNG, C.; KONG, H.; KRALJ, A.; MOONEY, S.; RAMACHANDRAN, S.; SIOW, M. L.. Sustainability and the tourism and hospitality workforce: A thematic analysis. Sustainability, v.8, n.8, p.809, 2016. DOI: https://doi.org/10.3390/su8080809

BOLUK, K. A.; CAVALIERE, C. T.; HIGGINS-DESBIOLLES, F.. A critical framework for interrogating the United Nations Sustainable Development Goals 2030 Agenda in tourism. Journal of Sustainable Tourism, v.27, n.7, p.847-864, 2019. DOI: https://doi.org/10.1080/09669582.2019.1619748

BUTLER, R. W.. Sustainable tourism: A state-of-the-art review. Tourism Geographies, v.1, n.1, p.7-25, 1999. DOI: http://doi.org/10.1080/14616689908721291

CHOI, H. C.; SIRAKAYA, E.. Sustainability indicators for managing community tourism. Tourism Management, v.27, n.6, p.1274-1289, 2006. DOI:

https://doi.org/10.1016/j.tourman.2005.05.018

ESLAMI, S.; KHALIFAH, Z.; MARDANI, A.; STREIMIKIENE, D.. Impact of Non-Economic Factors on Residents' support for Sustainable Tourism Development in Langkawi Island, Malaysia. Economics \& Sociology, v.11, n.4, p.181, 2018. DOI: http://doi.org/10.14254/2071-789X.2018/11-4/12

ETE. Ecological Tourism in Europe. Sustainable Tourism Development in UNESCO Designated Sites in South-Eastern Europe. Venice: UNESCO Office, 2009.

FARMAKI, A.; ALTINAY, L.; BOTTERILL, D.; HILKE, S.. Politics and sustainable tourism: The case of Cyprus. Tourism Management, v.47, p.178-190, 2015. DOI:

https://doi.org/10.1016/j.tourman.2014.09.019

GÖSSLING, S.. Global environmental consequences of tourism. Global Environmental Change, v.12, n.4, p.283-302, 2002. DOI: https://doi.org/10.1016/S0959-3780(02)00044-4

GLÄNZEL, W.. Bibliometrics as a research field: a course on theory and application of bibliometric indicators. 2003.

GRÁCIO, M. C. C.. Acoplamento bibliográfico e análise de cocitação: revisão teórico-conceitual. Revista Eletrônica de Biblioteconomia e Ciência da Informação, v.21, n.47, p.82, 2016. DOI: https://doi.org/10.5007/15182924.2016v21n47p82

HAK, T.; KOVANDA, J.; WEINZETTEL, J.. A method to assess the relevance of sustainability indicators: Application to the indicator set of the Czech Republic's Sustainable Development Strategy. Ecological Indicators, v.17, p.46-57, 2012. DOI: https://doi.org/10.1016/j.ecolind.2011.04.034

HALL, C.. Changing paradigms and global change: From sustainable to steady-state tourism. Tourism Recreation Research, v.35, n.2, p.131-143, 2010. DOI: https://doi.org/10.1080/02508281.2010.11081629

HALL, C.. Constructing sustainable tourism development: The 2030 agenda and the managerial ecology of sustainable tourism. Journal of Sustainable Tourism, p.1-17, 2019. DOI: https://doi.org/10.1080/09669582.2018.1560456

HARALAMBOPOULOS, N.; PIZAM, A.. Perceived impacts of tourism: The case of Samos. Annals of tourism Research, v.23, n.3, p.503-526, 1996. DOI:

https://doi.org/10.1016/0160-7383(95)00075-5

HIGGINS-DESBIOLLES, F.. Sustainable tourism: Sustaining tourism or something more?. Tourism management perspectives, v.25, p.157-160, 2018. DOI: https://doi.org/10.1016/j.tmp.2017.11.017

IMMACOLATA, V.. Agriculture, Rural Tourism and Circular Paradigm. Quality-Access to Success, 2018.

JACKSON, L. A.. Residents' perceptions of the impacts of special event tourism. Journal of Place Management and development, v.1, n.3, p.240-255, 2008. DOI: https://doi.org/10.1108/17538330810911244

KOENS, K.; POSTMA, A.; PAPP, B.. Is Overtourism overused? Understanding the impact of tourism in a city context. Sustainability, v.10, n.12, p.4384, 2018. DOI: https://doi.org/10.3390/su10124384

KLOPP, J. M.; PETRETTA, D. L.. The urban sustainable development goal: Indicators, complexity and the politics of measuring cities. Cities, v.63, p.92-97, 2017. DOI: https://doi.org/10.1016/j.cities.2016.12.019

LEW, A; NG, P. T; NI, C. C; WU, T. C.. Community sustainability and resilience: Similarities, differences and indicators. Tourism Geographies, v.18, n.1, p.18-27, 2016. DOI: http://doi.org/10.1080/14616688.2015.1122664

LUCAS, A.; VIERA, A. F. G.; PINTO, A. L.. Análise da produção científica sobre inteligência de negócios na web of science (wos). In: ENCONTRO NACIONAL DE PESQUISA EM PÓSGRADUAÇÃO EM CIÊNCIA DA INFORMAÇÃO, 16. Anais. João Pessoa: UFPB, 2015. p.1-17.

LUCAS, P.; LUDWIG, K.; KOK, M.; KRUITWAGEN, S. Sustainable Development in the Netherlands. PBL Netherlands Environmental Assessment Agency, 2016.

MILLER, G.; RATHOUSE, K.; SCARLES, C.; HOLMES, K.; TRIBE, J.. Public understanding of sustainable tourism. Annals of Tourism Research, v.37, n.3, p.627-645, 2010. DOI: https://doi.org/10.1016/j.annals.2009.12.002

MOLZ, J. G.. Social networking technologies and the moral economy of alternative tourism: The case of couchsurfing. Annals of tourism research, v.43, p.210-230, 2013. DOI: https://doi.org/10.1016/i.annals.2013.08.001

NUNKOO, R.; RAMKISSOON, H.. Developing a community support model for tourism. Annals of Tourism Research, v.38, n.3, p.964-988, 2011. DOI:

http://doi.org/10.1016/i.annals.2011.01.017

PAN, S. Y.; GAO, M.; KIM, H.; SHAH, K. J.; PEI, S. L.; CHIANG, P. C.. Advances and challenges in sustainable tourism toward a green economy. Science of the Total Environment, v.635, p.452-469, 2018. DOI: http://doi.org/10.1016/i.scitotenv.2018.04.134

QUEIROZ, D. G. C; MOURA, A. M. M.. Colaboração da Matemática brasileira na Web of Science (2004-2013). Revista Eletrônica de Biblioteconomia e Ciência da Informação, v.22, n.49, p.115-126, 2017. 
ROBU, V.; SOBOLEVSCHI, M. I.; PETCU, A. M.. Tourism: Vector of Sustainable Development: Quality-Access to Success Calitatea, v.20, Ed. S2, p.530-537, 2019.

SANTOS, A. S.; RIBEIRO, S. K.. The role of transport indicators to the improvement of local governance in Rio de Janeiro City: A contribution for the debate on sustainable future. Case Studies on Transport Policy, v.3, n.4, p.415-420, 2015. DOI: https://doi.org/10.1016/i.cstp.2015.08.006

SAARINEN, J.; ROGERSON, C. M.. Tourism and the millennium development goals perspectives beyond 2015. Tourism Geographies, v.16, n.1, p.23-30, 2014. DOI: https://doi.org/10.1080/14616688.2013.851269

SERAPHIN, H.; SHEERAN, P.; PILATO, M.. Over-tourism and the fall of Venice as a destination. Journal of Destination Marketing \& Management, v.9, p.374-376, 2018. DOI: https://doi.org/10.1016/j.jdmm.2018.01.011

SCHEYVENS, R.. Linking tourism to the sustainable development goals: a geographical perspective. Tourism Geographies, v.20, n.2, p.341-342, 2018. DOI: http://doi.org/10.1080/14616688.2018.1434818

SCHEYVENS, R.; BANKS, G.; HUGHES, E.. The Private Sector and the SDGs: The Need to Move Beyond 'Business as Usual'. Sustainable Development, v.24, n.6, p.371-382, 2016. DOI: https://doi.org/10.1002/sd.1623

SCOTT, D.; HALL, C. M.; GÖSSLING, S.. Global tourism vulnerability to climate change. Annals of Tourism Research, v.77, n.6, p.49-61, 2019. DOI:

https://doi.org/10.1016/j.annals.2019.05.007

SMALL, H.. Co-citation in the Scientific Literature: A New Measure of the Relationship Between Two Documents. Journal of the American Society for information Science, v.24, n.4, p.265-269, 1973. DOI: https://doi.org/10.1002/asi.4630240406
TANG, Z.. An integrated approach to evaluating the coupling coordination between tourism and the environment. Tourism Management, v.46, p.11-19, 2015. DOI: https://doi.org/10.1016/j.tourman.2014.06.001

UNWTO. United Nations World Tourism Organization. Discussion Paper on the Occasion of the International Year of Sustainable Tourism for Development 2017. Madrid: UNWTO, 2017.

UNWTO. United Nations World Tourism Organization. Overtourism? Understanding and Managing Urban Tourism Growth beyond Perceptions. Madrid: UNWTO, 2018.

VALENCIA, S. C.; SIMON, D.; CROESE, S.; NORDQVIST, J.; OLOKO, M.; SHARMA, T.; BUCK, N. T.; VERSACE, I.. Adapting the Sustainable Development Goals and the New Urban Agenda to the city level: Initial reflections from a comparative research project. International Journal of Urban Sustainable Development, v.11, n.1, p.4-23, 2019. DOI: https://doi.org/10.1080/19463138.2019.1573172

VAN ECK, N. J.; WALTMAN, L; DEKKER, R.; VAN DEN BERG, J.. A comparison of two techniques for bibliometric mapping: Multidimensional scaling and VOS. Journal of the American Society for Information Science and Technology, v.61, n.12, p.2405-2416, 2010. DOI: https://doi.org/10.1002/asi.21421

VAN ECK, N. J.; WALTMAN, L; DEKKER, R.; VAN DEN BERG, J.. CitNetExplorer: A new software tool for analyzing and visualizing citation networks. Journal of Informetrics, v.8, n.4, p.802-823, 2014. DOI: https://doi.org/10.1016/j.joi.2014.07.006

WEITZ, N.; PERSSON, Å.; NILSSON, M.; TENGGREN, S.. Sustainable Development Goals for Sweden: Insights on Setting a National Agenda. Stockholm Environment Institute, 2015.

A CBPC - Companhia Brasileira de Produção Científica (CNPJ: 11.221.422/0001-03) detém os direitos materiais desta publicação. Os direitos referem-se à publicação do trabalho em qualquer parte do mundo, incluindo os direitos às renovações, expansões e disseminações da contribuiç̃o, bem como outros direitos subsidiários. Todos os trabalhos publicados eletronicamente poderão posteriormente ser publicados em coletâneas impressas sob coordenação da Sustenere Publishing, da Companhia Brasileira de Produção Científica e seus parceiros autorizados. Os (as) autores (as) preservam os direitos autorais, mas não têm permissão para a publicação da contribuição em outro meio, impresso ou digital, em português ou em tradução. 